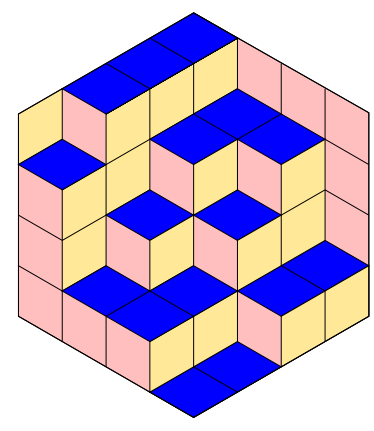

ALGEBRAIC COMBINATORICS

Shinsuke Iwao

Grothendieck polynomials and the boson-fermion correspondence

Volume 3, issue 5 (2020), p. 1023-1040.

<http://alco.centre-mersenne.org/item/ALCO_2020__3_5_1023_0>

(c) The journal and the authors, 2020.

Some rights reserved.

(c) BY This article is licensed under the

Creative Commons ATtribution 4.0 International LiCEnSE.

http://creativecommons.org/licenses/by/4.0/

Access to articles published by the journal Algebraic Combinatorics on the website http://alco.centre-mersenne.org/ implies agreement with the Terms of Use (http://alco.centre-mersenne.org/legal/).

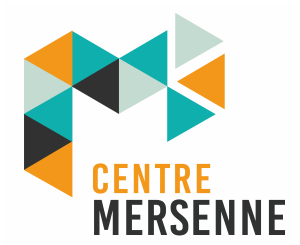

Algebraic Combinatorics is member of the Centre Mersenne for Open Scientific Publishing www.centre-mersenne.org 


\title{
Grothendieck polynomials and the boson-fermion correspondence
}

\author{
Shinsuke Iwao
}

\begin{abstract}
In this paper we study algebraic and combinatorial properties of symmetric Grothendieck polynomials and their dual polynomials by means of the boson-fermion correspondence. We show that these symmetric functions can be expressed as a vacuum expectation value of some operator that is written in terms of free-fermions. By using the free-fermionic expressions, we obtain alternative proofs of determinantal formulas and Pieri type formulas.
\end{abstract}

\section{INTRODUCTION}

Grothendieck polynomials $[13,14]$ are $K$-theoretic versions of Schubert polynomials that represent a Schubert variety in the $K$-theory of the flag variety. If it represents a Schubert class indexed by a Grassmannian permutation [2] (see also [5, § 10.6]), a Grothendieck polynomial is a symmetric polynomial in finitely many variables. Such symmetric Grothendieck polynomials are seen as $K$-theoretic analogs of Schur polynomials [16].

Let $\lambda=\left(\lambda_{1} \geqslant \cdots \geqslant \lambda_{\ell}>0\right)$ be a partition of a natural number, and $\beta$ be a parameter. A $(\beta-)$ Grothendieck polynomial ${ }^{(1)} G_{\lambda}\left(x_{1}, \ldots, x_{n}\right)(\ell \leqslant n)$ is a symmetric polynomial in $x_{1}, \ldots, x_{n}$ that is expressed as follows [7, 11, 22]:

$$
G_{\lambda}\left(x_{1}, \ldots, x_{n}\right)=\frac{\operatorname{det}\left(x_{i}^{\lambda_{j}+n-j}\left(1+\beta x_{i}\right)^{j-1}\right)_{1 \leqslant i, j \leqslant n}}{\prod_{1 \leqslant i<j \leqslant n}\left(x_{i}-x_{j}\right)} .
$$

There also exists the Jacobi-Trudi type identity $[11,15]$ :

$$
G_{\lambda}\left(x_{1}, \ldots, x_{n}\right)=\operatorname{det}\left(\sum_{m=0}^{\infty}\left(\begin{array}{c}
i-1 \\
m
\end{array}\right) \beta^{m} h_{\lambda_{i}-i+j+m}\left(x_{1}, \ldots, x_{n}\right)\right)_{1 \leqslant i, j \leqslant n},
$$

where $h_{i}\left(x_{1}, \ldots, x_{n}\right)$ is the $i$-th complete symmetric polynomial.

Recently, many authors have been studying connections between these " $K$ theoretic polynomials" and the theory of classical/quantum integrable systems.

Manuscript received 7th June 2019, revised 18th February 2020, accepted 25th February 2020. KEYWorDS. Symmetric Grothendieck polynomials, Boson-fermion correspondence.

ACKNOWLEdGements. This work is partially supported by JSPS Kakenhi Grant Number 19 K03605.

${ }^{(1)}$ The polynomial $G_{\lambda}\left(x_{1}, \ldots, x_{n}\right)$ is usually called the $\beta$-Grothendieck polynomial, which is a deformation of the ordinary Grothendieck polynomial introduced by Fomin-Kirillov [4]. The $\beta$ Grothendieck polynomial reduces to the Schur polynomial $s_{\lambda}\left(x_{1}, \ldots, x_{n}\right)$ when $\beta=0$, and to the ordinary Grothendieck polynomial when $\beta=-1$. We will drop the term " $\beta$-" throughout the paper to simplify the notation. 
In [18, 19], Motegi-Sakai showed that Grothendieck polynomials (and their generalizations) are derived from calculations of a wave function of quantum integrable systems such as TASEP and melting crystals. Nagai and the author of this paper [8] have reported that some class of dual stable Grothendieck polynomials are naturally obtained from tau functions of the relativistic Toda equation with unipotent eigenvalues.

The purpose of this paper is to present a new characterization of $G_{\lambda}\left(x_{1}, \ldots, x_{n}\right)$ by means of the boson-fermion correspondence (see, for example, $[9,10]$ ), which is a powerful algebraic tool in various fields such as symmetric polynomial theory, mathematical physics, integrable systems, etc. We show that the stable symmetric Grothendieck polynomial $G_{\lambda}\left(x_{1}, x_{2}, \ldots\right)$, that is, an infinite series of symmetric functions with $G_{\lambda}\left(x_{1}, \ldots, x_{n}, 0,0, \ldots\right)=G_{\lambda}\left(x_{1}, \ldots, x_{n}\right)$, can be expressed as a vacuum expectation value of some operator given in terms of free-fermions (Theorem 3.7). By using this expression, we derive a similar characterization of the dual stable Grothendieck polynomial (Section 4).

As an application of our presentation, we give new proofs of the following results, which have been given by previous researches:

(1) "Another" determinantal formula for Grothendieck polynomials (Proposition 3.9). This is a special case of the results by Hudson-Ikeda-MatsumuraNaruse [6].

(2) A determinantal formula for dual stable Grothendieck polynomials (Proposition 4.4 , which was originally given by $[12,21]$.

(3) $G_{\lambda}(x)$ expansion of symmetric polynomials of the form $s_{\lambda}(x) G_{\mu}(x)$ (Proposition 5.7).

(4) Pieri type formulas for dual stable Grothendieck polynomials (Section 6). Items (3-4) are special cases of the results given by Yeliussizov [22].

1.1. Organization of the Paper. In Section 2, we first give a brief review of the theory of free fermions $(\S 2.1-\S 2.3)$. Then we introduce two new operators $e^{\Theta}$ and $e^{\theta}$ and some simple lemmas in $\S 2.4$. In Section 3, we present a free-fermionic presentation of stable Grothendieck polynomials. For this, it is useful to consider a symmetric function $G_{\lambda}^{r}(x)$ (§ 3.1), which is "sufficiently near" to the Grothendieck polynomial $G_{\lambda}\left(x_{1}, \ldots, x_{r}\right)$ (see Proposition 3.1). We discuss the "stable limit" of the sequence $G_{\lambda}^{1}(x), G_{\lambda}^{2}(x), \ldots$. Since the sequence itself is not stable, the limit " $\lim _{r \rightarrow \infty} G_{\lambda}^{r}(x)$ " fails to be contained in $\Lambda$, the ring of symmetric functions. However, the limit will be defined properly in some completed space $\widehat{\Lambda} \supset \Lambda$ (see $\S 3.2)$. We show that the limit $\lim _{r \rightarrow \infty} G_{\lambda}^{r}(x)$ is expressed as a vacuum expectation value of a certain operator written in free-fermions, and is equal to the stable Grothendieck polynomial. "Another" determinantal formula for the stable Grothendieck polynomials is shown in $\S 3.5$. In Section 4, we obtain a similar free-fermionic presentation of dual stable Grothendieck polynomials. Their determinantal formula is also given (§ 4.3).

In Sections 5 and 6, we discuss Pieri type formulas for $K$-theoretic polynomials. By using our free-fermionic presentations, we define an action of noncommutative Schur polynomials [3] on the dense linear subspace of $\widehat{\Lambda}$ spanned by $\left\{G_{\lambda}(x) \mid \lambda\right.$ : partition $\}$. We also define their action on the subspace spanned by the family $\left\{g_{\lambda}(x) \mid \lambda\right.$ : partition $\}$, where $g_{\lambda}(x)$ is the dual stable Grothendieck polynomial. As a result, we derive the $G_{\lambda}(x)$ expansion of symmetric polynomials of the form $s_{\lambda}(x) G_{\mu}(x)$ (Proposition 5.7) and Pieri type formulas for dual stable Grothendieck polynomials (Section 6). 


\section{FreE FERMIONS}

2.1. Preliminaries. Let $k$ be a field of characteristic 0. (We will put $k=\mathbb{C}(\beta)$ in the sequel.) We consider a $k$-algebra $\mathcal{A}$ generated by free fermions $\psi_{n}, \psi_{n}^{*}(n \in \mathbb{Z})$ with the following anti-commutative relations:

$$
\left[\psi_{m}, \psi_{n}\right]_{+}=\left[\psi_{m}^{*}, \psi_{n}^{*}\right]_{+}=0, \quad\left[\psi_{m}, \psi_{n}^{*}\right]_{+}=\delta_{m, n},
$$

where $[A, B]_{+}=A B+B A$.

Let $|0\rangle,\langle 0|$ be vacuum vectors that satisfy

$$
\psi_{m}|0\rangle=\psi_{n}^{*}|0\rangle=0, \quad\langle 0| \psi_{n}=\langle 0| \psi_{m}^{*}=0, \quad m<0, n \geqslant 0 .
$$

The Fock space (over $k$ ) is the $k$-space $\mathcal{F}$ that is generated by vectors of the form

$$
\psi_{n_{1}} \psi_{n_{2}} \cdots \psi_{n_{r}} \psi_{m_{1}}^{*} \psi_{m_{2}}^{*} \cdots \psi_{m_{s}}^{*}|0\rangle,\left(r, s \geqslant 0, n_{1}>\cdots>n_{r} \geqslant 0>m_{s}>\cdots>m_{1}\right) \text {. }
$$

We also consider the $k$-space $\mathcal{F}^{*}$ that is generated by vectors

$\langle 0| \psi_{m_{s}} \cdots \psi_{m_{2}} \psi_{m_{1}} \psi_{n_{r}}^{*} \cdots \psi_{n_{2}}^{*} \psi_{n_{1}}^{*},\left(r, s \geqslant 0, n_{1}>\cdots>n_{r} \geqslant 0>m_{s}>\cdots>m_{1}\right)$.

The vectors of the form (4) are linearly independent over $k$. This fact can be checked by identifying $\mathcal{F}$ with an infinite wedge presentation of a Clifford algebra $[10, \S 4$ and $\S 5.2]$. See Remark 2.1 below. Similarly, the vectors of the form (5) are proved to be linearly independent.

Using the anti-commutative relations (3) repeatedly, we find $|v\rangle \in \mathcal{F} \Rightarrow \psi_{n}|v\rangle \in \mathcal{F}$ and $\psi_{n}^{*}|v\rangle \in \mathcal{F}$. Hence $\mathcal{F}$ is a left $\mathcal{A}$-module. We can also check that $\mathcal{F}^{*}$ is a right $\mathcal{A}$-module.

REMARK 2.1. Let $V=\bigoplus_{i \in \mathbb{Z}} k \cdot v_{i}$ be a $k$-space with a fixed basis $\left\{v_{i} \mid i \in \mathbb{Z}\right\}$ and $\bigwedge^{\infty} V$ be the infinite wedge space. For $m \in \mathbb{Z}$, consider the subspace $F^{(m)} \subset \Lambda^{\infty} V$ generated by all vectors of the form

$$
v_{i_{1}} \wedge v_{i_{2}} \wedge \cdots \quad\left(i_{1}>i_{2}>\cdots, i_{k}=-k+m \text { for } k \gg 1\right) .
$$

Set $F:=\bigoplus_{m \in \mathbb{Z}} F^{(m)}$. We can define the actions of $\psi_{j}, \psi_{j}^{*}$ on $F[10, \S 5.2]$ by

$$
\begin{aligned}
\psi_{j}\left(v_{i_{1}} \wedge v_{i_{2}} \wedge \cdots\right)=v_{j} \wedge v_{i_{1}} \wedge v_{i_{2}} \wedge \cdots, & \\
\psi_{j}^{*}\left(v_{i_{1}} \wedge v_{i_{2}} \wedge \cdots\right)=f\left(v_{i_{1}}\right) v_{i_{2}} \wedge v_{i_{3}} \wedge v_{i_{4}} \wedge & \cdots-f\left(v_{i_{2}}\right) v_{i_{1}} \wedge v_{i_{3}} \wedge v_{i_{4}} \wedge \cdots \\
& +f\left(v_{i_{3}}\right) v_{i_{1}} \wedge v_{i_{2}} \wedge v_{i_{4}} \wedge \cdots-\cdots,
\end{aligned}
$$

where $f: V \rightarrow k$ is the $k$-linear map that sends $v_{j} \mapsto 1$ and $v_{t} \mapsto 0(t \neq j)$. We can check that they satisfy the relation (3). By sending the vacuum vector $|0\rangle$ to the element $v_{-1} \wedge v_{-2} \wedge v_{-3} \wedge \cdots \in F$, we obtain a left $\mathcal{A}$-module homomorphism $\mathcal{F} \rightarrow F$, which is in fact an isomorphism. Rigorous statements and proofs of these facts can be found in the textbooks $[10, \S 5]$ and $[17, \S 4]$. The review article $[1, \S 2]$ by Alexandrov and Zabrobin is helpful to understand the free-fermion formalism.

For an integer $m$, we define the shifted vacuum vectors $|m\rangle \in \mathcal{F}$ and $\langle m| \in \mathcal{F}^{*}$ by

$$
|m\rangle= \begin{cases}\psi_{m-1} \psi_{m-2} \cdots \psi_{0}|0\rangle, & m \geqslant 0 \\ \psi_{m}^{*} \cdots \psi_{-2}^{*} \psi_{-1}^{*}|0\rangle, & m<0\end{cases}
$$

and

$$
\langle m|= \begin{cases}\langle 0| \psi_{0}^{*} \psi_{1}^{*} \ldots \psi_{m-1}^{*}, & m \geqslant 0, \\ \langle 0| \psi_{-1} \psi_{-2} \ldots \psi_{m}, & m<0 .\end{cases}
$$

We define an anti-algebra involution on $\mathcal{A}$ by

$$
{ }^{*}: \mathcal{A} \rightarrow \mathcal{A} ; \quad \psi_{n} \leftrightarrow \psi_{n}^{*},
$$


that is, a $k$-linear isomorphism with $(a b)^{*}=b^{*} a^{*}$ and $\left(a^{*}\right)^{*}=a$. Further, we have an isomorphism of $k$-spaces

$$
\omega: \mathcal{F} \rightarrow \mathcal{F}^{*}, \quad X|0\rangle \mapsto\langle 0| X^{*} .
$$

The vacuum expectation value is the unique $k$-bilinear map

$$
\mathcal{F}^{*} \otimes_{k} \mathcal{F} \rightarrow k, \quad\langle w|\otimes| v\rangle \mapsto\langle w \mid v\rangle
$$

that is determined by $\langle 0 \mid 0\rangle=1,\left(\langle w| \psi_{n}\right)|v\rangle=\langle w|\left(\psi_{n}|v\rangle\right)$, and $\left(\langle w| \psi_{n}^{*}\right)|v\rangle=$ $\langle w|\left(\psi_{n}^{*}|v\rangle\right)$. For any expression $X$, we write $\left.\langle w|X| v\rangle:=(\langle w| X)|v\rangle=\langle w|(|X| v\rangle\right)$. The expectation value $\langle 0|X| 0\rangle$ is often abbreviated as $\langle X\rangle$.

REMARK 2.2. The existence of the vacuum expectation value can be checked by using the infinite wedge presentation as follows. Let $(\cdot, \cdot)$ be the non-degenerate symmetric $k$-bilinear form on $F$ where the set of vectors (6) are orthonormal. From (7), we have $\left(\psi_{i}|v\rangle,|w\rangle\right)=\left(|v\rangle, \psi_{i}^{*}|w\rangle\right)$, which means $\psi_{i}$ and $\psi_{i}^{*}$ are adjoint to each other. The vacuum expectation value is defined by $\langle w \mid v\rangle:=(\omega(|w\rangle),|v\rangle)$.

2.2. WiCK's THEOREM. In many cases, vacuum expectation values can be calculated by using the following Wick's theorem.

Theorem 2.3 (Wick's theorem (see $[1, \S 2],[17$, Exercise 4.2$])$ ). Let $\left\{m_{1}, \ldots, m_{r}\right\}$ and $\left\{n_{1}, \ldots, n_{r}\right\}$ be sets of integers. Then we have

$$
\left\langle\psi_{m_{1}} \cdots \psi_{m_{r}} \psi_{n_{r}}^{*} \cdots \psi_{n_{1}}^{*}\right\rangle=\operatorname{det}\left(\left\langle\psi_{m_{i}} \psi_{n_{j}}^{*}\right\rangle\right)_{1 \leqslant i, j \leqslant r}
$$

For sets of integers $m=\left\{m_{1}, \ldots, m_{r}\right\}, n=\left\{n_{1}, \ldots, n_{s}\right\}$ with $m_{1}>\cdots>m_{r}$, $n_{1}>\cdots>n_{s}$, we write

$$
\delta_{m, n}= \begin{cases}1, & r=s \text { and } m_{i}=n_{i} \text { for all } i \\ 0, & \text { otherwise. }\end{cases}
$$

Corollary 2.4. Let $m=\left\{m_{1}, \ldots, m_{r}\right\}, n=\left\{n_{1}, \ldots, n_{s}\right\}$ be sets of integers with $m_{1}>\cdots>m_{r}>-r$ and $n_{1}>\cdots>n_{s}>-s$. Then,

$$
\left\langle-r\left|\psi_{m_{r}}^{*} \cdots \psi_{m_{1}}^{*} \psi_{n_{1}} \cdots \psi_{n_{s}}\right|-s\right\rangle=\delta_{m, n} .
$$

2.3. The BOSON-FERMION CORRESPONDENCE. Let : • : be the normal ordering (see $[1, \S 2],[17, \S 5.2]$ ) of free-fermions defined as follows: all annihilation operators $\left(\psi_{m}(m<0)\right.$ and $\left.\psi_{n}^{*}(n \geqslant 0)\right)$ are moved to the right, and an appropriate sign factor $( \pm 1)$ is multiplied. For example, we have : $\psi_{1} \psi_{1}^{*}:=\psi_{1} \psi_{1}^{*}$ and $: \psi_{1}^{*} \psi_{1}:=-\psi_{1} \psi_{1}^{*}$.

For $m \in \mathbb{Z}$, we define an operator $a_{m}$ as $a_{m}=\sum_{k \in \mathbb{Z}}: \psi_{k} \psi_{k+m}^{*}:$ on $\mathcal{F}$. The operator $a_{m}$ satisfies the following commutative relations

$$
\left[a_{m}, a_{n}\right]=m \delta_{m+n, 0}, \quad\left[a_{m}, \psi_{n}\right]=\psi_{n-m}, \quad\left[a_{m}, \psi_{n}^{*}\right]=-\psi_{n+m}^{*},
$$

where $[A, B]=A B-B A$. For proofs of these facts, see $[17, \S 5.3]$. We also note that, if $|v\rangle \leftrightarrow\langle w|$ under the involution $\omega$, we have $a_{n}|v\rangle \leftrightarrow\langle w| a_{-n}$.

Let $x_{1}, x_{2}, \ldots$ be formal independent variables. We set

$$
H(x)=\sum_{n>0} \frac{p_{n}(x)}{n} a_{n}, \quad p_{n}(x)=x_{1}^{n}+x_{2}^{n}+\cdots,(\text { the } n \text {-th power sum }),
$$

which satisfies the commutative relation

$$
e^{H(x)} \psi_{n}=\left(\sum_{i=0}^{\infty} h_{i}(x) \psi_{n-i}\right) e^{H(x)},
$$

where $h_{i}(x)\left(i \in \mathbb{Z}_{\geqslant 0}\right)$ is the $i$-th complete symmetric function. 
Theorem 2.5 ([17, Lemma 9.5] (also see [10, Theorem 6.1])). Let $\lambda=\left(\lambda_{1} \geqslant \cdots \geqslant\right.$ $\left.\lambda_{\ell}>0\right)$ be a partition of length $\ell$. We set $\lambda_{\ell+1}=\lambda_{\ell+2}=\cdots=\lambda_{r}=0$ for $r \geqslant \ell$. Then we have

$$
s_{\lambda}(x)=\left\langle 0\left|e^{H(x)} \psi_{\lambda_{1}-1} \psi_{\lambda_{2}-2} \cdots \psi_{\lambda_{r}-r}\right|-r\right\rangle=\operatorname{det}\left(h_{\lambda_{i}-i+j}(x)\right)_{1 \leqslant i, j \leqslant r},
$$

where $s_{\lambda}(x)$ is the Schur function.

2.4. Operators $e^{\Theta}$ AND $e^{\theta}$. Let $\psi(z)$ and $\psi^{*}(z)$ be the generating functions of $\psi_{n}$ and $\psi_{n}^{*}$ with a formal variable $z$ :

$$
\psi(z)=\sum_{n \in \mathbb{Z}} \psi_{n} z^{n}, \quad \psi^{*}(z)=\sum_{n \in \mathbb{Z}} \psi_{n}^{*} z^{n} .
$$

Note that $\left[a_{n}, \psi(z)\right]=z^{n} \psi(z)$ and $\left[a_{n}, \psi^{*}(z)\right]=-z^{-n} \psi^{*}(z)$.

We now introduce two important operators

$$
\Theta=\beta a_{-1}-\frac{\beta^{2}}{2} a_{-2}+\frac{\beta^{3}}{3} a_{-3}-\cdots \text { and } \theta=\beta a_{1}-\frac{\beta^{2}}{2} a_{2}+\frac{\beta^{3}}{3} a_{3}-\cdots .
$$

Since

$$
e^{X} Y e^{-X}=Y+\operatorname{ad}_{X} \cdot Y+\frac{\left(\operatorname{ad}_{X}\right)^{2}}{2} \cdot Y+\cdots,
$$

$\left(\operatorname{ad}_{X} \cdot Y=[X, Y]\right)$, we have the following equations

$$
e^{\Theta} \psi(z) e^{-\Theta}=\left(1+\beta z^{-1}\right) \psi(z), \quad e^{\theta} \psi(z) e^{-\theta}=(1+\beta z) \psi(z) .
$$

We give a list of lemmas that are useful in the following sections.

LEMMA 2.6. $e^{\Theta} \psi_{n} e^{-\Theta}=\psi_{n}+\beta \psi_{n+1}, e^{\theta} \psi_{n} e^{-\theta}=\psi_{n}+\beta \psi_{n-1}$.

LEMMA 2.7. $e^{H(x)} e^{\Theta}=\prod_{i=1}^{\infty}\left(1+\beta x_{i}\right)^{-1} \cdot e^{\Theta} e^{H(x)}$.

LEMMA 2.8. $e^{H(x)} \psi(z)=\left(\sum_{i=0}^{\infty} h_{i}(x) z^{i}\right) \psi(z) e^{H(x)}$.

Lemmas 2.6-2.8 can be shown from (9-10) by straightforward calculations.

LEMMA 2.9. For $m \in \mathbb{Z}$ and $s \geqslant 1$, we have

$$
\psi_{m-1} e^{\Theta} \psi_{m-2} e^{\Theta} \cdots \psi_{m-s} e^{\Theta}=\psi_{m-1} \psi_{m-2} \cdots \psi_{m-s} e^{s \Theta}
$$

Proof. We prove the lemma by induction on $s \geqslant 1$. If $s=1$, the equation is trivial. For $s \geqslant 1$, we have by induction hypothesis

$$
\begin{aligned}
\psi_{m-1} e^{\Theta} & \psi_{m-2} e^{\Theta} \cdots \psi_{m-s} e^{\Theta} \psi_{m-s-1} e^{\Theta} \\
& =\psi_{m-1} e^{\Theta}\left(\psi_{m-2} \psi_{m-3} \ldots \psi_{m-s-1} e^{s \Theta}\right) \\
& =\psi_{m-1}\left(\psi_{m-2}+\beta \psi_{m-1}\right)\left(\psi_{m-3}+\beta \psi_{m-2}\right) \ldots\left(\psi_{m-s-1}+\beta \psi_{m-s}\right) e^{(s+1) \Theta} .
\end{aligned}
$$

Since $\psi_{m-1}^{2}=\psi_{m-2}^{2}=\cdots=\psi_{m-s}^{2}=0$, the last expression can be rewritten as

$$
\psi_{m-1} \psi_{m-2} \psi_{m-3} \ldots \psi_{m-s-1} e^{(s+1) \Theta} \text {. }
$$

LEMma 2.10. For $m \in \mathbb{Z}$ and $s \geqslant 1$, we have

$$
\psi_{m-1} e^{\Theta} \psi_{m-2} e^{\Theta} \cdots \psi_{m-s} e^{\Theta} \psi_{m-s} e^{\Theta}=(-\beta)^{s} \psi_{m} e^{\Theta} \psi_{m-1} e^{\Theta} \cdots \psi_{m-s+1} e^{\Theta} \psi_{m-s} e^{\Theta} .
$$

Proof. We prove the lemma by induction on $s$. When $s=1$, it follows that

$$
\begin{aligned}
\psi_{m-1} e^{\Theta} \psi_{m-1} e^{\Theta} & =e^{\Theta}\left(\psi_{m-1}-\beta \psi_{m}+\beta^{2} \psi_{m+1}-\cdots\right) \psi_{m-1} e^{\Theta} \\
& =e^{\Theta}\left(-\beta \psi_{m}+\beta^{2} \psi_{m+1}-\cdots\right) \psi_{m-1} e^{\Theta} \\
& =(-\beta) \psi_{m} e^{\Theta} \psi_{m-1} e^{\Theta}
\end{aligned}
$$


from $\psi_{m-1}^{2}=0$. For general $s$, we have

$$
\begin{aligned}
\psi_{m-1} e^{\Theta} \cdots \psi_{m-s-1} e^{\Theta} \psi_{m-s} e^{\Theta} \psi_{m-s} e^{\Theta} & \\
& =(-\beta) \psi_{m-1} e^{\Theta} \cdots \psi_{m-s-1} e^{\Theta} \psi_{m-s-1} e^{\Theta} \psi_{m-s} e^{\Theta} \\
& =(-\beta)^{s} \psi_{m} e^{\Theta} \cdots \psi_{m-s-2} e^{\Theta} \psi_{m-s-1} e^{\Theta} \psi_{m-s} e^{\Theta}
\end{aligned}
$$

by induction hypothesis.

CoRollary 2.11. Set $X(n):=\psi_{n_{1}-1} e^{\Theta} \psi_{n_{2}-2} e^{\Theta} \cdots \psi_{n_{r}-r} e^{\Theta}$ for $n=\left(n_{1}, \ldots, n_{r}\right)$. Assume $n_{1}-1 \geqslant n_{2}-2 \geqslant \cdots \geqslant n_{r}-r$. Then the following equation holds:

$$
X(n)=(-\beta)^{|\bar{n}|-|n|} \cdot X(\bar{n}),
$$

where $\bar{n}_{j}=\max \left[n_{j}, n_{j+1}, \ldots, n_{r}\right],|n|=\sum_{j=1}^{r} n_{j}$, and $|\bar{n}|=\sum_{j=1}^{r} \bar{n}_{j}$.

Similarly, we have:

Lemma 2.12. For $m \in \mathbb{Z}$, it follows that

$$
\psi_{m} e^{-\theta} \psi_{m}=(-\beta) \psi_{m} e^{-\theta} \psi_{m-1}
$$

Proof. Since $\psi_{m}^{2}=0$, we have $\psi_{m} e^{-\theta} \psi_{m}=\psi_{m}\left(\psi_{m}-\beta \psi_{m-1}+\beta^{2} \psi_{m+2}-\cdots\right) e^{-\theta}=$ $\psi_{m}\left(-\beta \psi_{m-1}+\beta^{2} \psi_{m+2}-\cdots\right) e^{-\theta}=(-\beta) \psi_{m} e^{-\theta} \psi_{m-1}$.

Corollary 2.13. Set $x(n):=\psi_{n_{1}-1} e^{-\theta} \psi_{n_{2}-2} e^{-\theta} \cdots \psi_{n_{r}-r} e^{-\theta}$ for $n=\left(n_{1}, \ldots, n_{r}\right)$. Assume $n_{1}-1 \geqslant n_{2}-2 \geqslant \cdots \geqslant n_{r}-r$. Then the following equation holds:

$$
x(n)=(-\beta)^{|n|-|\underline{n}|} \cdot x(\underline{n}),
$$

where $\underline{n}_{j}=\min \left[n_{1}, \ldots, n_{j}\right]$.

\section{Stable Grothendieck polynomial $G_{\lambda}(x)$}

3.1. Definition of $G_{\lambda}^{r}(x)$. Let $\lambda=\left(\lambda_{1} \geqslant \cdots \geqslant \lambda_{\ell}>0\right)$ be a partition. For $r \geqslant \ell$, we put $\lambda_{\ell+1}=\lambda_{\ell+2}=\cdots=\lambda_{r}=0$. Let $G_{\lambda}^{r}(x)$ denote the symmetric function that is defined by

$$
G_{\lambda}^{r}(x):=\left\langle 0\left|e^{H(x)} \psi_{\lambda_{1}-1} e^{\Theta} \psi_{\lambda_{2}-2} e^{\Theta} \cdots \psi_{\lambda_{r}-r} e^{\Theta} \cdot e^{-r \Theta}\right|-r\right\rangle .
$$

This expression is rewritten as

$$
G_{\lambda}^{r}(x)=\left\langle 0\left|e^{H(x)} \psi_{\lambda_{1}-1} e^{\Theta} \psi_{\lambda_{2}-2} e^{\Theta} \cdots \psi_{\lambda_{\ell}-\ell} e^{\Theta} \cdot \psi_{-\ell-1} \cdots \psi_{-r} \cdot e^{-\ell \Theta}\right|-r\right\rangle
$$

by using Lemma 2.9 .

Proposition 3.1. If $\ell(\lambda) \leqslant n \leqslant r$, we have

$$
G_{\lambda}\left(x_{1}, \ldots, x_{n}\right)=G_{\lambda}^{r}\left(x_{1}, \ldots, x_{n}, 0,0, \ldots\right) .
$$

Proof. Let us consider the generating function

$$
\Psi\left(z_{1}, \ldots, z_{r}\right):=\left\langle 0\left|e^{H(x)} \psi\left(z_{1}\right) e^{\Theta} \psi\left(z_{2}\right) e^{\Theta} \cdots \psi\left(z_{r}\right) e^{\Theta} \cdot e^{-r \Theta}\right|-r\right\rangle
$$

of $G_{\lambda}^{r}(x)$. Set $A_{i}=e^{(i-1) \Theta} \psi\left(z_{i}\right) e^{-(i-1) \Theta}=\left(1+\beta z_{i}^{-1}\right)^{i-1} \psi\left(z_{i}\right)$. From Wick's theorem (Theorem 2.3), it follows that

$$
\Psi\left(z_{1}, \ldots, z_{r}\right)=\left\langle 0\left|e^{H(x)} A_{1} A_{2} \ldots A_{r}\right|-r\right\rangle=\operatorname{det}\left(\left\langle 0\left|e^{H(x)} A_{i} e^{-H(x)} \psi_{-j}^{*}\right| 0\right\rangle\right)_{1 \leqslant i, j \leqslant r} .
$$

By substituting $e^{H(x)} A_{i} e^{-H(x)}=\left(1+\beta z_{i}^{-1}\right)^{i-1}\left(\sum_{m=0}^{\infty} h_{m}(x) z_{i}^{m}\right) \psi\left(z_{i}\right)$, which follows from Lemma 2.8, we have

$$
\begin{aligned}
\Psi\left(z_{1}, \ldots, z_{r}\right) & =\operatorname{det}\left(\left(1+\beta z_{i}^{-1}\right)^{i-1}\left(\sum_{m=0}^{\infty} h_{m}(x) z_{i}^{m}\right)\left\langle 0\left|\psi\left(z_{i}\right) \psi_{-j}^{*}\right| 0\right\rangle\right)_{1 \leqslant i, j \leqslant r} \\
& =\operatorname{det}\left(\left(1+\beta z_{i}^{-1}\right)^{i-1}\left(\sum_{m=0}^{\infty} h_{m}(x) z_{i}^{m}\right) z_{i}^{-j}\right)_{1 \leqslant i, j \leqslant r} .
\end{aligned}
$$


Comparing the coefficients of $z_{1}^{\lambda_{1}-1} z_{2}^{\lambda_{2}-2} \cdots z_{r}^{\lambda_{r}-r}$ on the both sides, we obtain

$$
G_{\lambda}^{r}(x)=\operatorname{det}\left(\sum_{m=0}^{\infty}\left(\begin{array}{c}
i-1 \\
m
\end{array}\right) \beta^{m} h_{\lambda_{i}-i+j+m}(x)\right)_{1 \leqslant i, j \leqslant r} .
$$

From (2), we have the desired result.

3.2. The Completed RING $\widehat{\Lambda}$. Put $k=\mathbb{C}(\beta)$. Let $\Lambda$ be the $k$-algebra of symmetric functions $[16, \S \mathrm{I} .2]$ in $x_{1}, x_{2}, \ldots$ In this section we give a brief review on the completed $\operatorname{ring} \widehat{\Lambda} \supset \Lambda$.

Let $M_{n}(n \geqslant 1)$ be the $k$-subspace of $\Lambda$ that is expressed as

$$
M_{n}:=\left\{\sum_{i=1}^{N} c_{\lambda_{i}} s_{\lambda_{i}}(x) ; N \geqslant 0, \lambda_{1}, \ldots, \lambda_{N} \text { are partitions, } c_{\lambda_{i}} \in k, \ell\left(\lambda_{i}\right) \geqslant n,\right\},
$$

where $\ell(\lambda)$ is the length of a partition $\lambda$. Since $M_{n} \supset M_{n+1}$, an inverse system

$$
\Lambda / M_{1} \leftarrow \Lambda / M_{2} \leftarrow \Lambda / M_{3} \leftarrow \cdots
$$

of $k$-spaces exists. Let $\widehat{\Lambda}:=\lim \left(\Lambda / M_{n}\right)$ be the inverse limit. Note that there exists a natural inclusion $\Lambda \hookrightarrow \widehat{\Lambda}$.

It is convenient to introduce a $k$-linear topology on $\Lambda$ where the family $\left\{M_{n}\right\}_{n=1,2, \ldots}$ forms an open neighborhood base at 0 . In terms of this topology, the inclusion $\Lambda \hookrightarrow \widehat{\Lambda}$ can be viewed as a completion of the topological space $\Lambda$. Note that

$$
f(x) \in M_{n+1} \Longleftrightarrow f\left(x_{1}, \ldots, x_{n}, 0,0, \ldots\right)=0 .
$$

Moreover, $\widehat{\Lambda}$ is indeed a topological $k$-algebra, over which the multiplication is also continuous.

LEMMA 3.2. If $n_{1}, \ldots, n_{r}>-r$, then $\left\langle 0\left|e^{H(x)} \psi_{n_{1}} \ldots \psi_{n_{r}}\right|-r\right\rangle \in M_{r}$.

Proof. It follows from Theorem 2.5.

It is known that there exists a unique element $G_{\lambda}(x) \in \widehat{\Lambda}$, which is called the stable Grothendieck polynomial [4], that satisfies the equation

$$
G_{\lambda}\left(x_{1}, \ldots, x_{n}\right)=G_{\lambda}\left(x_{1}, \ldots, x_{n}, 0,0, \ldots\right)
$$

for any $n$. From Proposition 3.1, we have

$$
G_{\lambda}(x)-G_{\lambda}^{r}(x) \in M_{n+1} \quad \text { for any } \ell(\lambda) \leqslant n \leqslant r,
$$

which implies the fact that ' $G_{\lambda}(x)$ and $G_{\lambda}^{r}(x)$ are sufficiently near.' From (12), by putting $n=r$, we have $G_{\lambda}(x)-G_{\lambda}^{r}(x) \in M_{r+1}$. As a subset of the topological space $\widehat{\Lambda}$, the sequence $G_{\lambda}^{1}(x), G_{\lambda}^{2}(x), \cdots \in \widehat{\Lambda}$ converges to $G_{\lambda}(x)$. We simply write this fact as

$$
G_{\lambda}(x)=\lim _{r \rightarrow \infty} G_{\lambda}^{r}(x)
$$

3.3. REMARKS ON ELEMENTS OF $\widehat{\Lambda}$. We will often interested in symmetric functions of the form

$$
\left\langle 0\left|e^{H(x)} \psi_{m_{1}} \ldots \psi_{m_{r}} e^{s \Theta}\right|-r\right\rangle
$$

where $r, s \geqslant 0$ and $m_{1}, \ldots, m_{r}>-r$. In general, such symmetric function cannot be contained in $\Lambda$. If fact, if $r=0$ and $s=1$, we have

$$
\left\langle 0\left|e^{H(x)} e^{\Theta}\right| 0\right\rangle=\prod_{i=1}^{\infty}\left(1+\beta x_{i}\right) \cdot\left\langle 0\left|e^{\Theta} e^{H(x)}\right| 0\right\rangle=\prod_{i=1}^{\infty}\left(1+\beta x_{i}\right) \in \widehat{\Lambda} \backslash \Lambda .
$$


We can check that, if we substitute $x_{n+1}=x_{n+2}=\cdots=0$, this function reduces to a symmetric polynomial in $n$ variables. The following lemma states that the same is true for any symmetric function of the form (14).

LemMA 3.3. Let $H\left(x_{1}, \ldots, x_{n}\right):=\left.H(x)\right|_{x_{n+1}=x_{n+2}=\cdots=0}$. Then

$$
f\left(x_{1}, \ldots, x_{n}\right):=\left\langle 0\left|e^{H\left(x_{1}, \ldots, x_{n}\right)} \psi_{m_{1}} \ldots \psi_{m_{r}} e^{s \Theta}\right|-r\right\rangle
$$

is a symmetric polynomial in $x_{1}, \ldots, x_{n}$.

Proof. Let $X_{-r}:=\left(\begin{array}{c}s \\ 1\end{array}\right) \beta \psi_{-r}+\left(\begin{array}{c}s \\ 2\end{array}\right) \beta^{2} \psi_{-r+1}+\cdots+\left(\begin{array}{c}s \\ s\end{array}\right) \beta^{s} \psi_{-r-1+s}$. Since $e^{s \Theta} \psi_{-r-1}=$ $\left(\psi_{-r-1}+X_{-r}\right) e^{s \Theta}$, we have

$$
\begin{aligned}
& \left\langle 0\left|e^{H\left(x_{1}, \ldots, x_{n}\right)} \psi_{m_{1}} \ldots \psi_{m_{r}} e^{s \Theta}\right|-r\right\rangle \\
& =\left\langle 0\left|e^{H\left(x_{1}, \ldots, x_{n}\right)} \psi_{m_{1}} \ldots \psi_{m_{r}}\right|-r\right\rangle \\
& \quad+\left\langle 0\left|e^{H\left(x_{1}, \ldots, x_{n}\right)} \psi_{m_{1}} \ldots \psi_{m_{r}} X_{-r}\right|-r-1\right\rangle \\
& \quad+\left\langle 0\left|e^{H\left(x_{1}, \ldots, x_{n}\right)} \psi_{m_{1}} \ldots \psi_{m_{r}}\left(\psi_{-r-1}+X_{-r}\right) X_{-r-1}\right|-r-2\right\rangle \\
& \quad+\left\langle 0\left|e^{H\left(x_{1}, \ldots, x_{n}\right)} \psi_{m_{1}} \ldots \psi_{m_{r}}\left(\psi_{-r-1}+X_{-r}\right)\left(\psi_{-r-2}+X_{-r-1}\right) X_{-r-2}\right|-r-3\right\rangle \\
& \quad+\cdots .
\end{aligned}
$$

Because

$$
\left\langle 0\left|e^{H\left(x_{1}, \ldots, x_{n}\right)} \psi_{m_{1}} \ldots \psi_{m_{r}}\left(\psi_{-r-1}+X_{-r}\right) \cdots\left(\psi_{-r-t}+X_{-r-t+1}\right) X_{-r-t}\right|-r-t-1\right\rangle
$$

is 0 if $r+t>n$ (see Lemma 3.2), the right hand side on (16) is in fact a polynomial.

From Lemma 3.3, $f\left(x_{1}, \ldots, x_{n}\right)$ in (15) determines a unique element of $\Lambda / M_{n+1}$. Because $f\left(x_{1}, \ldots, x_{n}\right)=f\left(x_{1}, \ldots, x_{n}, 0\right)$ for any $n$, there uniquely exists an element $f(x)=f\left(x_{1}, x_{2}, \ldots\right) \in \widehat{\Lambda}$ which satisfies $f\left(x_{1}, \ldots, x_{n}\right)=f\left(x_{1}, \ldots, x_{n}, 0,0, \ldots\right)$. In other words, we have

$$
f(x)=\left\langle 0\left|e^{H(x)} \psi_{m_{1}} \ldots \psi_{m_{r}} e^{s \Theta}\right|-r\right\rangle .
$$

The expression (16) implies that:

Proposition 3.4. $\left\langle 0\left|e^{H(x)} \psi_{m_{1}} \ldots \psi_{m_{r}}\left(e^{s \Theta}-1\right)\right|-r\right\rangle \in M_{r+1}$.

The following lemma will be useful in the next section.

LEMMA 3.5. Let $m_{1}, \ldots, m_{\ell}$ be a sequence of integers. Then

$$
\begin{aligned}
\langle 0| e^{H(x)} \psi_{m_{1}} \ldots \psi_{m_{\ell}} \psi_{-\ell-1} & e^{\Theta} \psi_{-\ell-2} e^{\Theta} \cdots \psi_{-r} e^{\Theta}|-r\rangle \\
& =\left\langle 0\left|e^{H(x)} \psi_{m_{1}} \ldots \psi_{m_{\ell}} \psi_{-\ell-1} \psi_{-\ell-2} \cdots \psi_{-r} e^{(r-\ell) \Theta}\right|-r\right\rangle \\
& =\left\langle 0\left|e^{H(x)} \psi_{m_{1}} \ldots \psi_{m_{\ell}} \psi_{-\ell-1} \psi_{-\ell-2} \cdots \psi_{-r}\right|-r\right\rangle \\
& =\left\langle 0\left|e^{H(x)} \psi_{m_{1}} \ldots \psi_{m_{\ell}}\right|-\ell\right\rangle .
\end{aligned}
$$

Proof. The first equality follows from Lemma 2.9. Let

$$
Y_{-r}:=\left(\begin{array}{c}
r-\ell \\
1
\end{array}\right) \beta \psi_{-r}+\left(\begin{array}{c}
r-\ell \\
2
\end{array}\right) \beta^{2} \psi_{-r+1}+\cdots+\left(\begin{array}{c}
r-\ell \\
r-\ell
\end{array}\right) \beta^{r-\ell} \psi_{-\ell-1} .
$$

Because $\psi_{-\ell-1} \psi_{-\ell-2} \cdots \psi_{-r-t} Y_{-r-t}=0$ for any $t \geqslant 0$, the equation (16) is now simplified as

$$
\begin{aligned}
\left\langle 0\left|e^{H\left(x_{1}, \ldots, x_{n}\right)} \psi_{m_{1}} \ldots \psi_{m_{\ell}} \psi_{-\ell-1} \psi_{-\ell-2} \cdots \psi_{-r} e^{(r-\ell) \Theta}\right|-r\right\rangle & \\
& =\left\langle 0\left|e^{H\left(x_{1}, \ldots, x_{n}\right)} \psi_{m_{1}} \ldots \psi_{m_{\ell}} \psi_{-\ell-1} \psi_{-\ell-2} \cdots \psi_{-r}\right|-r\right\rangle
\end{aligned}
$$

which implies the second equality. The third equality is obvious. 
REMARK 3.6. If $s^{\prime}<0$, the expression $\left\langle 0\left|e^{H(x)} \psi_{m_{1}} \ldots \psi_{m_{r}} e^{s^{\prime} \Theta}\right|-r\right\rangle$ does not determine an element of $\widehat{\Lambda}$. In fact, if $r=0$ and $s^{\prime}=-1$, the expression is rewritten as $\left\langle 0\left|e^{H(x)} e^{-\Theta}\right| 0\right\rangle=\prod_{i=1}^{\infty}\left(1+\beta x_{i}\right)^{-1}$, which is not contained in $\widehat{\Lambda}$.

3.4. Free-FERMionic EXPRESSion of $G_{\lambda}(x)$. Let us consider the symmetric function

$$
\bar{G}_{\lambda}(x):=\left\langle 0\left|e^{H(x)} \psi_{\lambda_{1}-1} e^{\Theta} \psi_{\lambda_{2}-2} e^{\Theta} \cdots \psi_{\lambda_{\ell}-\ell} e^{\Theta}\right|-\ell\right\rangle .
$$

Note that the symmetric functions $G_{\lambda}^{r}(x)$ and $\bar{G}_{\lambda}(x)$ are quite similar but different. Assume $r \geqslant \ell$. From Lemma 3.5, their difference is expressed as

$$
\bar{G}_{\lambda}(x)-G_{\lambda}^{r}(x)=\left\langle 0\left|e^{H(x)} \psi_{\lambda_{1}-1} e^{\Theta} \cdots \psi_{\lambda_{\ell}-\ell} e^{\Theta} \psi_{-\ell-1} \cdots \psi_{-r} e^{-\ell \Theta}\left(e^{\ell \Theta}-1\right)\right|-r\right\rangle .
$$

From this equation and Proposition 3.4, we have

$$
\bar{G}_{\lambda}(x)-G_{\lambda}^{r}(x) \in M_{r+1},
$$

which implies that the sequence $\left\{G_{\lambda}^{r}(x)\right\}_{r=1,2, \ldots}$ converges to $\bar{G}_{\lambda}(x)$ in $\widehat{\Lambda}$. It follows from (13) that

$$
\bar{G}_{\lambda}(x)=\lim _{r \rightarrow \infty} G_{\lambda}^{r}(x)=G_{\lambda}(x) .
$$

In other words, we have:

THEOREM 3.7.

$$
G_{\lambda}(x)=\left\langle 0\left|e^{H(x)} \psi_{\lambda_{1}-1} e^{\Theta} \psi_{\lambda_{2}-2} e^{\Theta} \cdots \psi_{\lambda_{\ell}-\ell} e^{\Theta}\right|-\ell\right\rangle .
$$

3.5. "Another" Determinant FORMula FOR $G_{\lambda}(x)$. We often write $G_{n}(x)=$ $G_{(n)}(x)$, where $(n)$ is a partition of length 1 .

Proposition 3.8. We have

$$
\sum_{n \in \mathbb{Z}} G_{n}(x) z^{n}=\frac{1}{1+\beta z^{-1}} \prod_{i=1}^{\infty} \frac{1+\beta x_{i}}{1-x_{i} z},
$$

where $G_{n}(x)=\left\langle 0\left|e^{H(x)} \psi_{n-1} e^{\Theta}\right|-1\right\rangle$ and $\left(1+\beta z^{-1}\right)^{-1}=\sum_{n=0}^{\infty}(-\beta)^{n} z^{-n}$.

Proof.

$$
\begin{aligned}
\sum_{n \in \mathbb{Z}} G_{n}(x) z^{n} & =\left\langle 0\left|e^{H(x)} \psi(z) z e^{\Theta}\right|-1\right\rangle=\left(1+\beta z^{-1}\right)^{-1}\left\langle 0\left|e^{H(x)} e^{\Theta} \psi(z) z\right|-1\right\rangle \\
& =\left(1+\beta z^{-1}\right)^{-1} \prod_{i=1}^{\infty}\left(1+\beta x_{i}\right)\left\langle 0\left|e^{H(x)} \psi(z) z\right|-1\right\rangle \\
& =\left(1+\beta z^{-1}\right)^{-1} \prod_{i=1}^{\infty}\left(1+\beta x_{i}\right) \cdot\left(\sum_{i=0}^{\infty} h_{i}(x) z^{i}\right) \\
& =\frac{1}{1+\beta z^{-1}} \prod_{i=1}^{\infty} \frac{1+\beta x_{i}}{1-x_{i} z} .
\end{aligned}
$$

Let $\mathcal{G}(z):=\sum_{n \in \mathbb{Z}} G_{n}(x) z^{n}$. From the proof of Proposition 3.8, we derive the commutative relation

$$
e^{H(x)} e^{-\Theta} \psi(z) e^{\Theta} e^{-H(x)}=\prod_{i=1}^{\infty}\left(1+\beta x_{i}\right)^{-1} \cdot \mathcal{G}(z) \psi(z) .
$$

We consider the formal function

$$
\bar{\Psi}\left(z_{1}, \ldots, z_{r}\right):=\left\langle 0\left|e^{H(x)} \psi\left(z_{1}\right) e^{\Theta} \cdots \psi\left(z_{r}\right) e^{\Theta}\right|-r\right\rangle,
$$

which is a generating function of $G_{\lambda}(x)$.

Proposition 3.9 ([6], see also [20]). We have

$$
G_{\lambda}(x)=\operatorname{det}\left(\sum_{m=0}^{\infty}\left(\begin{array}{c}
i-r \\
m
\end{array}\right) \beta^{m} G_{\lambda_{i}-i+j+m}(x)\right)_{1 \leqslant i, j \leqslant r} .
$$


Proof. Let $B_{i}:=e^{-(r-i+1) \Theta} \psi\left(z_{i}\right) e^{(r-i+1) \Theta}$. Applying Wick's theorem (Theorem 2.3) to the generating function (19) gives

$$
\begin{aligned}
\bar{\Psi}\left(z_{1}, \ldots, z_{r}\right) & =\left\langle 0\left|e^{H(x)} e^{r \Theta} B_{1} B_{2} \ldots B_{r}\right|-r\right\rangle \\
& =\prod_{l=1}^{\infty}\left(1+\beta x_{i}\right)^{r}\left\langle 0\left|e^{H(x)} B_{1} B_{2} \ldots B_{r}\right|-r\right\rangle \\
& =\prod_{l=1}^{\infty}\left(1+\beta x_{i}\right)^{r} \cdot \operatorname{det}\left(\left\langle 0\left|e^{H(x)} B_{i} e^{-H(x)} \psi_{-j}^{*}\right| 0\right\rangle\right)_{1 \leqslant i, j \leqslant r} .
\end{aligned}
$$

Since

$$
\begin{aligned}
e^{H(x)} B_{i} e^{-H(x)} & =\left(1+\beta z_{i}^{-1}\right)^{-(r-i)} e^{H(x)} e^{-\Theta} \psi\left(z_{i}\right) e^{\Theta} e^{-H(x)} \\
& =\left(\prod_{l=1}^{\infty}\left(1+\beta x_{l}\right)^{-1}\right) \cdot\left(1+\beta z_{i}^{-1}\right)^{-(r-i)} \mathcal{G}\left(z_{i}\right) \psi\left(z_{i}\right)
\end{aligned}
$$

(see (18)), we have

$$
\begin{aligned}
\bar{\Psi}\left(z_{1}, \ldots, z_{r}\right) & =\operatorname{det}\left(\left(1+\beta z_{i}^{-1}\right)^{-(r-i)} \mathcal{G}\left(z_{i}\right)\left\langle 0\left|\psi\left(z_{i}\right) \psi_{-j}^{*}\right| 0\right\rangle\right)_{1 \leqslant i, j \leqslant r} \\
& =\operatorname{det}\left(\left(1+\beta z_{i}^{-1}\right)^{-(r-i)} \mathcal{G}\left(z_{i}\right) z_{i}^{-j}\right)_{1 \leqslant i, j \leqslant r}
\end{aligned}
$$

Comparing the coefficients of $z_{1}^{\lambda_{1}-1} \cdots z_{r}^{\lambda_{r}-r}$ on the both sides, we obtain the desired equation.

\section{Dual stable Grothendieck polynomial $g_{\lambda}(x)$}

4.1. Definition. For a partition $\lambda=\left(\lambda_{1} \geqslant \lambda_{2} \geqslant \cdots \geqslant \lambda_{\ell}>0\right)$ and $\lambda_{\ell+1}=\cdots=$ $\lambda_{r}=0$, we set

$$
g_{\lambda}(x):=\left\langle 0\left|e^{H(x)} \psi_{\lambda_{1}-1} e^{-\theta} \psi_{\lambda_{2}-2} e^{-\theta} \cdots \psi_{\lambda_{r}-r} e^{-\theta}\right|-r\right\rangle .
$$

Note the the definition of $g_{\lambda}(x)$ does not depend on the choice of $r \geqslant \ell$ because of the equation $|-r\rangle=\psi_{-r-1}|-r-1\rangle=\psi_{-r-1} e^{-\theta}|-r-1\rangle$.

4.2. Proof of The DuAlity. The Hall inner product $\langle\cdot, \cdot\rangle: \Lambda \times \Lambda \rightarrow k$ is the nondegenerate $k$-bilinear form that satisfies $\left\langle s_{\lambda}(x), s_{\mu}(x)\right\rangle=\delta_{\lambda, \mu}$. The bilinear form can be uniquely extended to the bilinear form $\widehat{\Lambda} \times \Lambda \rightarrow k$ continuously.

Let $X=\psi_{n_{1}} \cdots \psi_{n_{r}}$ and $Y=\psi_{m_{1}} \cdots \psi_{m_{s}}$. If two symmetric functions $f(x)$ and $g(x)$ are expressed as $f(x)=\left\langle 0\left|e^{H(x)} X\right|-r\right\rangle$ and $g(x)=\left\langle 0\left|e^{H(x)} Y\right|-s\right\rangle$, their Hall inner product can be calculated by using the formula

$$
\langle f(x), g(x)\rangle=\left\langle-r\left|X^{*} Y\right|-s\right\rangle,
$$

which is obtained from Corollary 2.4.

Proposition 4.1. We have

$$
\left\langle G_{\lambda}(x), g_{\mu}(x)\right\rangle=\delta_{\lambda, \mu} .
$$

This means that $g_{\lambda}(x)$ is nothing but the dual stable Grothendieck polynomial.

To prove Proposition 4.1, it suffices to show (20) $\left\langle-r\left|e^{\theta} \psi_{\lambda_{r}-r}^{*} \cdots e^{\theta} \psi_{\lambda_{2}-2}^{*} e^{\theta} \psi_{\lambda_{1}-1}^{*} \psi_{\mu_{1}-1} e^{-\theta} \psi_{\mu_{2}-2} e^{-\theta} \cdots \psi_{\mu_{s}-s} e^{-\theta}\right|-s\right\rangle=\delta_{\lambda, \mu}$.

For this, we need the following two lemmas.

LEMMA 4.2. If $N>n_{1}, \ldots, n_{s}$, then $\psi_{N}^{*} \psi_{n_{1}} e^{-\theta} \psi_{n_{2}} e^{-\theta} \cdots \psi_{n_{s}} e^{-\theta}|-s\rangle=0$.

Proof. As $e^{-\theta} \psi_{n} e^{\theta}=\psi_{n}-\beta \psi_{n-1}+\beta^{2} \psi_{n-2}-\cdots$, the vector $\psi_{n_{1}} e^{-\theta} \cdots \psi_{n_{s}} e^{-\theta}|-s\rangle$ must be a linear combination of vectors of the form

$$
\psi_{n_{1}^{\prime}} \cdots \psi_{n_{s}^{\prime}}|-s\rangle, \quad N>n_{1}^{\prime}, \ldots, n_{s}^{\prime} .
$$

Since $\left[\psi_{m}^{*}, \psi_{n}\right]_{+}=0$ for $m \neq n$, we obtain the desired result. 
LEMMA 4.3. If $M>m_{1}>\cdots>m_{r} \geqslant-s$, then $\langle-s| e^{\theta} \psi_{m_{r}}^{*} \ldots e^{\theta} \psi_{m_{1}}^{*} \psi_{M}=0$.

Proof. We prove by induction on $r \geqslant 0$. If $r=0$ and $M \geqslant-s$, the equation $\langle-s| \psi_{M}=$ 0 is obvious. Next assume $r \geqslant 1$. Since $e^{\theta} \psi_{m_{1}}^{*} \psi_{M}=-\left(\psi_{M}+\beta \psi_{M-1}\right) e^{\theta} \psi_{m_{1}}^{*}$, we have

$$
\langle-s| e^{\theta} \psi_{m_{r}}^{*} \cdots e^{\theta} \psi_{m_{2}}^{*} e^{\theta} \psi_{m_{1}}^{*} \psi_{M}=-\langle-s| e^{\theta} \psi_{m_{r}}^{*} \cdots e^{\theta} \psi_{m_{2}}^{*}\left(\psi_{M}+\beta \psi_{M-1}\right) e^{\theta} \psi_{m_{1}}^{*} .
$$

Because $M-1>m_{2}$, this equals to 0 by induction hypothesis.

Proof of Proposition 4.1. Let

$$
C:=\left\langle-r\left|e^{\theta} \psi_{\lambda_{r}-r}^{*} \cdots e^{\theta} \psi_{\lambda_{2}-2}^{*} e^{\theta} \psi_{\lambda_{1}-1}^{*} \psi_{\mu_{1}-1} e^{-\theta} \psi_{\mu_{2}-2} e^{-\theta} \cdots \psi_{\mu_{s}-s} e^{-\theta}\right|-s\right\rangle .
$$

If $\lambda_{1}>\mu_{1}$, then $C=0$ from Lemma 4.2. If $\lambda_{1}<\mu_{1}$, then $C=0$ from Lemma 4.3. Assume $\lambda_{1}=\mu_{1}$. Since $\psi_{\lambda_{1}-1}^{*} \psi_{\mu_{1}-1}=1-\psi_{\mu_{1}-1} \psi_{\lambda_{1}-1}^{*}, C$ is rewritten as

$$
C=\left\langle-r\left|e^{\theta} \psi_{\lambda_{r}-r}^{*} \cdots e^{\theta} \psi_{\lambda_{2}-2}^{*} \psi_{\mu_{2}-2} e^{-\theta} \cdots \psi_{\mu_{s}-s} e^{-\theta}\right|-s\right\rangle
$$

by using Lemma 4.2 again. Repeating this procedure, we conclude that $C=\delta_{\lambda, \mu}$.

\subsection{Determinant Formula FOR $g_{\lambda}(x)$.}

Proposition 4.4 ([12, 21]). We have

$$
g_{\lambda}(x)=\operatorname{det}\left(\sum_{m=0}^{\infty}\left(\begin{array}{c}
1-i \\
m
\end{array}\right) \beta^{m} h_{\lambda_{i}-i+j-m}(x)\right)_{1 \leqslant i, j \leqslant r} .
$$

Proof. Let

$$
\Phi\left(z_{1}, \ldots, z_{r}\right)=\left\langle 0\left|e^{H(x)} \psi\left(z_{1}\right) e^{-\theta} \psi\left(z_{2}\right) e^{-\theta} \cdots \psi\left(z_{r}\right) e^{-\theta}\right|-r\right\rangle .
$$

We put $D_{i}:=e^{-(i-1) \theta} \psi\left(z_{i}\right) e^{(i-1) \theta}=\left(1+\beta z_{i}\right)^{-(i-1)} \psi\left(z_{i}\right)$. Since $e^{\theta}|-r\rangle=|-r\rangle$, we have

$$
\begin{aligned}
\Phi\left(z_{1}, \ldots, z_{r}\right) & =\left\langle 0\left|e^{H(x)} D_{1} D_{2} \ldots D_{r}\right|-r\right\rangle \\
& =\operatorname{det}\left(\left\langle 0\left|e^{H(x)} D_{i} e^{-H(x)} \psi_{-j}^{*}\right| 0\right\rangle\right)_{1 \leqslant i, j \leqslant r} \\
& =\operatorname{det}\left(\left(1+\beta z_{i}\right)^{-(i-1)}\left(\sum_{m=0}^{\infty} h_{m}(x) z_{i}^{m}\right)\left\langle 0\left|\psi\left(z_{i}\right) \psi_{-j}^{*}\right| 0\right\rangle\right)_{1 \leqslant i, j \leqslant r} \\
& =\operatorname{det}\left(\left(1+\beta z_{i}\right)^{-(i-1)}\left(\sum_{m=0}^{\infty} h_{m}(x) z_{i}^{m}\right) z_{i}^{-j}\right)_{1 \leqslant i, j \leqslant r} .
\end{aligned}
$$

Comparing the coefficients of $z_{1}^{\lambda_{1}-1} \cdots z_{r}^{\lambda_{r}-r}$ on the both sides gives the desired expression.

\section{Application 1: $G_{\lambda}(x)$-EXPANSiOn OF SYMmetric FUnCTIONS}

In the following two sections, we present a new method of deriving Pieri type formulas for $K$-theoretic polynomials. We will define an action of non-commutative Schur polynomials [3] on Grothendieck polynomials and dual stable Grothendieck polynomials by using their free-fermionic presentations. This enables us to express symmetric functions of the form $s_{\lambda}(x) G_{\mu}(x)$ (resp. $s_{\lambda}(x) g_{\mu}(x)$ ) as a linear combination of Grothendieck polynomials (resp. dual stable Grothendieck polynomials). 


\section{1. $\beta$-Twisted SChur OPERATORS. Let}

$$
\mathfrak{X}:=\bigoplus_{\lambda} \mathbb{Q}[\beta] \cdot \lambda
$$

be the $\mathbb{Q}[\beta]$-module freely generated by all partitions $\lambda$. We define a linear operator $u_{i}: \mathfrak{X} \rightarrow \mathfrak{X},(i>0)$, which we will call a $\beta$-twisted Schur operator. For any sequence $n=\left(n_{1}, \ldots, n_{\ell}\right)$, we let $\bar{n}=\left(\bar{n}_{1}, \ldots, \bar{n}_{\ell}\right)$ denote the smallest partition that satisfies $n_{i} \leqslant \bar{n}_{i}$ for all $i$. We have $\bar{n}_{i}=\max \left[n_{i}, n_{i+1}, \ldots, n_{\ell}\right]$.

We write $\mathbf{e}_{i}=(0, \ldots, \stackrel{i}{v}, \ldots, 0)$.

DeFINITION 5.1. Let $u_{i}: \mathfrak{X} \rightarrow \mathfrak{X}$ be the linear operator that acts on a partition $\lambda$ as

$$
u_{i} \cdot \lambda=(-\beta)^{\left|\overline{\lambda+\mathbf{e}_{i}}\right|-\left|\lambda+\mathbf{e}_{i}\right|} \cdot \overline{\lambda+\mathbf{e}_{i}} .
$$

EXAMPLE 5.2.

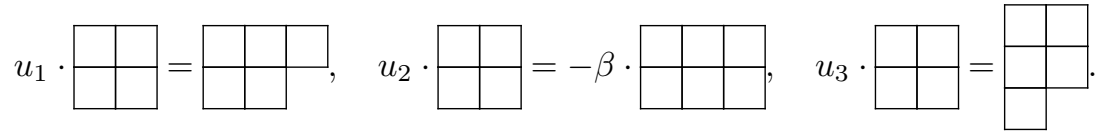

EXAmple 5.3. Since $\overline{\left(\overline{\lambda+\mathbf{e}_{i}}\right)+\mathbf{e}_{j}}=\overline{\lambda+\mathbf{e}_{i}+\mathbf{e}_{j}}$ for $i<j$, the action of operators of the form $u_{i_{1}} \cdots u_{i_{r}}\left(i_{1}>\cdots>i_{r}\right)$ is expressed as

$$
u_{i_{1}} \cdots u_{i_{r}} \cdot \lambda=(-\beta)^{\left|\overline{\lambda+\mathbf{e}_{i_{1}}+\cdots+\mathbf{e}_{i_{r}}}\right|-\left|\lambda+e_{i_{1}}+\cdots+e_{i_{r}}\right|} \cdot \overline{\lambda+\mathbf{e}_{i_{1}}+\cdots+\mathbf{e}_{i_{r}}} .
$$

LEMma 5.4. The $\beta$-twisted Schur operators satisfy the following commutative relations.

$$
\begin{array}{ll}
u_{i} u_{k} u_{j}=u_{k} u_{i} u_{j}, & i \leqslant j<k, \\
u_{j} u_{i} u_{k}=u_{j} u_{k} u_{i}, & i<j \leqslant k .
\end{array}
$$

Proof. They are directly checked by seeing their actions on the basis.

Equation (22) in Lemma 5.4 are often called the Knuth relation. It was proved by Fomin and Greene [3] that the theory of non-commutative Schur functions is applicable to any set of operators that satisfies the Knuth relation.

5.2. Non-COMmutative Schur functions. Let $T$ be a semi-standard tableau, or a tableau [5]. The column word $w_{T}$ of $T$ is the sequence of numbers obtained by reading the entries of $T$ from bottom to top in each column, starting in the left column and moving to the right. For example, $w_{T}=3215344$ for $T=$\begin{tabular}{|l|l|l|l}
\hline 1 & 3 & 4 & 4 \\
\hline 2 & 5 & &
\end{tabular} . We define the monomial $u^{T}$ as

$$
u^{T}:=u_{w_{T}(1)} u_{w_{T}(2)} \cdots u_{w_{T}(N)} .
$$

Definition 5.5 (Non-commutative Schur function). For a partition $\lambda$, we define $s_{\lambda}\left(u_{1}, \ldots, u_{n}\right)$ by

$$
s_{\lambda}\left(u_{1}, \ldots, u_{n}\right):=\sum_{\begin{array}{c}
T \text { is of shape } \lambda, \\
\text { each entry of } T \text { is } \leqslant n .
\end{array}} u^{T} .
$$

If $\lambda=\left(1^{i}\right)=(\overbrace{1, \ldots, 1}^{i})$, the operator $e_{i}\left(u_{1}, \ldots, u_{n}\right):=s_{\left(1^{i}\right)}\left(u_{1}, \ldots, u_{n}\right)$ is called the $i$-th non-commutative elementary symmetric polynomial. If $\lambda=(i), h_{i}\left(u_{1}, \ldots, u_{n}\right):=$ $s_{(i)}\left(u_{1}, \ldots, u_{n}\right)$ is called the $i$-th non-commutative complete symmetric polynomial.

The following proposition is given by Fomin-Greene [3]. 
Proposition 5.6 (Fundamental properties of non-commutative Schur functions). Let $u_{1}, \ldots, u_{n}$ be a set of non-commutative operators with the Knuth relation (22). Let $\Lambda_{n}(u)$ denote the ring of non-commutative Schur functions in $u_{1}, \ldots, u_{n}$. Then $\Lambda_{n}(u)$ is commutative; that is, we have

$$
s_{\lambda}\left(u_{1}, \ldots, u_{n}\right) s_{\mu}\left(u_{1}, \ldots, u_{n}\right)=s_{\mu}\left(u_{1}, \ldots, u_{n}\right) s_{\lambda}\left(u_{1}, \ldots, u_{n}\right)
$$

for any $\lambda, \mu$. Moreover, the (commutative) ring $\Lambda_{n}(u)$ is generated by all noncommutative elementary polynomials $e_{i}\left(u_{1}, \ldots, u_{n}\right)$ for $i=0,1, \ldots, n$. As a polynomial of $e_{i}\left(u_{1}, \ldots, u_{n}\right)$ 's, the non-commutative Schur polynomial $s_{\lambda}\left(u_{1}, \ldots, u_{n}\right)$ is expressed as

$$
s_{\lambda}\left(u_{1}, \ldots, u_{n}\right)=\operatorname{det}\left(e_{\lambda_{i}-i+j}\left(u_{1}, \ldots, u_{n}\right)\right)_{1 \leqslant i, j \leqslant r}, \quad \ell(\lambda) \leqslant n \leqslant r,
$$

which is exactly same as the Jacobi-Trudi formula for ordinal Schur polynomials.

5.3. $u_{n}$-ACTION ON Grothendieck polynomials. Let $\pi: \mathfrak{X} \rightarrow \widehat{\Lambda}$ is the $\mathbb{Q}[\beta]$ linear map that sends a partition $\lambda$ to $G_{\lambda}(x)$. The following proposition provides an algorithm to express a product $s_{\lambda}(x) G_{\mu}(x)$ as a linear combination of Grothendieck polynomials.

Proposition 5.7. For $\ell(\lambda), \ell(\mu) \leqslant r$, the equation

$$
s_{\lambda}(x) G_{\mu}(x) \equiv \pi\left(s_{\lambda}\left(u_{1}, \ldots, u_{r}\right) \cdot \mu\right) \bmod M_{r+1}
$$

holds. In other words, we have

$$
s_{\lambda}\left(x_{1}, \ldots, x_{n}\right) G_{\mu}\left(x_{1}, \ldots, x_{n}\right)=\left.\pi\left(s_{\lambda}\left(u_{1}, \ldots, u_{r}\right) \cdot \mu\right)\right|_{x_{n+1}=x_{n+2}=\cdots=0}
$$

for $\ell(\lambda), \ell(\mu) \leqslant n \leqslant r$.

Proof. From Proposition 5.6, it suffices to prove

$$
e_{i}(x) G_{\mu}(x) \equiv \pi\left(e_{i}\left(u_{1}, \ldots, u_{r}\right) \cdot \mu\right) \bmod M_{r+1}
$$

for $i=0,1, \ldots, r$. Write $f(x)=\left\langle 0\left|e^{H(x)} \psi_{n_{1}-1} e^{\Theta} \cdots \psi_{n_{r}-r} e^{\Theta}\right|-r\right\rangle$ for a sequence of integers $n_{1}, \ldots, n_{r}$. Since $e^{H(x)} a_{-i} e^{-H(x)}=a_{-i}+p_{i}(x),\left[a_{-i}, \psi_{m}\right]=\psi_{m+i}$, and $\left[a_{-i}, e^{\Theta}\right]=0$, we have

$$
\begin{aligned}
p_{i}(x) f(x)= & \left\langle 0\left|e^{H(x)} a_{-i} \psi_{n_{1}-1} e^{\Theta} \cdots \psi_{n_{r}-r} e^{\Theta}\right|-r\right\rangle \\
= & \sum_{j=1}^{r}\left\langle 0\left|e^{H(x)} \psi_{n_{1}-1} e^{\Theta} \cdots \psi_{n_{j}-j+i} e^{\Theta} \cdots \psi_{n_{r}-r} e^{\Theta}\right|-r\right\rangle \\
& \quad+\left\langle 0\left|e^{H(x)} \psi_{n_{1}-1} e^{\Theta} \cdots \psi_{n_{r}-r} e^{\Theta} a_{-i}\right|-r\right\rangle .
\end{aligned}
$$

This equation implies

(24) $p_{i}(x) f(x) \equiv \sum_{j=1}^{r}\left\langle 0\left|e^{H(x)} \psi_{n_{1}-1} e^{\Theta} \cdots \psi_{n_{j}-j+i} e^{\Theta} \cdots \psi_{n_{r}-r} e^{\Theta}\right|-r\right\rangle \quad \bmod M_{r+1}$

because $\left\langle 0\left|e^{H(x)} \psi_{n_{1}-1} e^{\Theta} \cdots \psi_{n_{r}-r} e^{\Theta} a_{-i}\right|-r\right\rangle$ is contained in $M_{r+1}$ by Lemma 3.2 . Let $E_{i}\left(p_{1}, \ldots, p_{i}\right)$ be the polynomial in $p_{1}, \ldots, p_{i}$ that satisfies

$$
e_{i}(x)=E_{i}\left(p_{1}(x), \ldots, p_{i}(x)\right) .
$$

From (24), we show that the product $e_{i}(x) f(x)$ satisfies

$$
\begin{aligned}
e_{i}(x) f(x) & =\left\langle 0\left|e^{H(x)} E_{i}\left(a_{-1}, \ldots, a_{-i}\right) \psi_{n_{1}-1} e^{\Theta} \cdots \psi_{n_{r}-r} e^{\Theta}\right|-r\right\rangle \\
\equiv & \sum_{1 \leqslant m_{1}<\cdots<m_{i} \leqslant r}\langle 0| e^{H(x)} \psi_{n_{1}-1} e^{\Theta} \cdots \psi_{n_{m_{1}}-m_{1}+1} e^{\Theta} \cdots \psi_{n_{m_{i}}-m_{i}+1} e^{\Theta} \\
\cdots \psi_{n_{r}-r} e^{\Theta}|-r\rangle & \bmod M_{r+1} .
\end{aligned}
$$


For any sequence $n=\left(n_{1}, \ldots, n_{r}\right)$, write

$$
Y(n)=\left\langle 0\left|e^{H(x)} \psi_{n_{1}-1} e^{\Theta} \cdots \psi_{n_{r}-r} e^{\Theta}\right|-r\right\rangle .
$$

(Note that $\pi(\lambda)=Y(\lambda)$ if $\lambda$ is a partition.) Substituting $f(x)=G_{\mu}(x)$ to the above equation gives

$$
e_{i}(x) G_{\mu}(x) \equiv \sum_{1 \leqslant m_{1}<\cdots<m_{i} \leqslant r} Y\left(\mu+\mathbf{e}_{m_{1}}+\cdots+\mathbf{e}_{m_{i}}\right) \quad \bmod M_{r+1} .
$$

From Corollary 2.11 and (21), this implies

$$
\begin{aligned}
& e_{i}(x) G_{\mu}(x) \equiv \sum_{1 \leqslant m_{1}<\cdots<m_{i} \leqslant r}(-\beta)^{\mid \overline{\mu+\mathbf{e}_{m_{1}}+\cdots+\mathbf{e}_{m_{i}}}}|-| \mu+e_{m_{1}}+\cdots+e_{m_{i}} \mid Y\left(\overline{\mu+\mathbf{e}_{m_{1}}+\cdots+\mathbf{e}_{m_{i}}}\right) \\
& =\sum_{1 \leqslant m_{1}<\cdots<m_{i} \leqslant r} \pi\left(u_{m_{i}} \ldots u_{m_{1}} \cdot \mu\right) \\
& =\pi\left(e_{i}\left(u_{1}, \ldots, u_{r}\right) \cdot \mu\right) \bmod M_{r+1} \text {. }
\end{aligned}
$$

From Proposition 5.7, we find a systematic way to express symmetric polynomials of the form $s_{\lambda}\left(x_{1}, \ldots, x_{n}\right) G_{\mu}\left(x_{1}, \ldots, x_{n}\right)$ as a linear combination of $G_{\lambda}\left(x_{1}, \ldots, x_{n}\right)$ 's. See the examples below.

EXAMPLE 5.8. Since

$$
h_{2}\left(u_{1}, u_{2}\right)=s \square \square\left(u_{1}, u_{2}\right)=u_{1} u_{1}+u_{1} u_{2}+u_{2} u_{2},
$$

we have

$$
\begin{aligned}
& h_{2}\left(x_{1}, x_{2}\right) \cdot G_{\varnothing}\left(x_{1}, x_{2}\right)=G_{\square \square}\left(x_{1}, x_{2}\right)-\beta G_{\square}\left(x_{1}, x_{2}\right)+\beta^{2} G_{\square}\left(x_{1}, x_{2}\right) \text {, } \\
& h_{2}\left(x_{1}, x_{2}\right) \cdot G_{\square}\left(x_{1}, x_{2}\right)=G_{\square \square}\left(x_{1}, x_{2}\right)+G_{\square} \square^{\left.\left(x_{1}, x_{2}\right)-\beta G_{\square} \square^{\left(x_{1}\right.}, x_{2}\right)}, \\
& \left.h_{2}\left(x_{1}, x_{2}\right) \cdot G_{\square \square}\left(x_{1}, x_{2}\right)=G_{\square}{ }_{\square} \square^{\left(x_{1}, x_{2}\right)+G_{\square}^{\square} \square^{\square}}\left(x_{1}, x_{2}\right)+G_{\square}{ }^{\left(x_{1}\right.}, x_{2}\right) \text {, etc. }
\end{aligned}
$$

EXAMPLE 5.9. Let $\lambda=\square$. All Young tableaux of the shape $\lambda$ with entries at most 3 are given as follows:

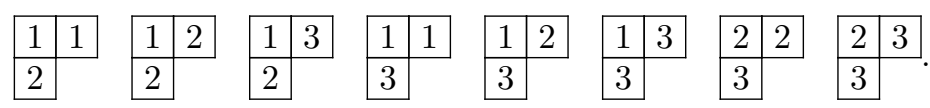

We therefore have

$$
\begin{aligned}
& \stackrel{s}{\square}{ }^{\left(u_{1}, u_{2}, u_{3}\right)} \\
& \quad=u_{2} u_{1} u_{1}+u_{2} u_{1} u_{2}+u_{2} u_{1} u_{3}+u_{3} u_{1} u_{1}+u_{3} u_{1} u_{2}+u_{3} u_{1} u_{3}+u_{3} u_{2} u_{2}+u_{3} u_{2} u_{3} .
\end{aligned}
$$

By using this, we obtain $\left(s_{\lambda}=s_{\lambda}\left(x_{1}, x_{2}, x_{3}\right), G_{\lambda}=G_{\lambda}\left(x_{1}, x_{2}, x_{3}\right)\right)$

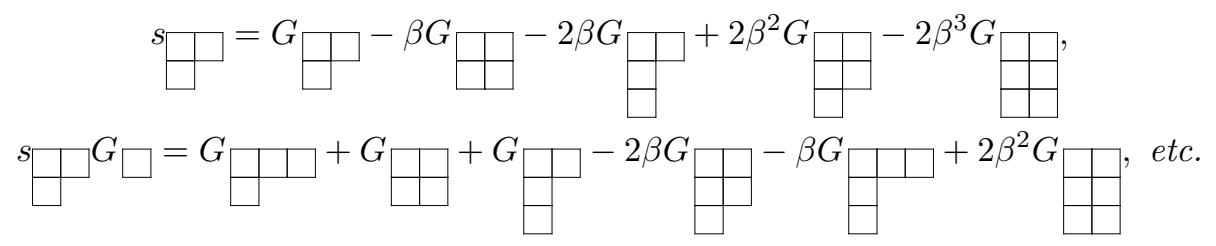




\section{Application 2: Pieri type formula for $g_{\lambda}$}

Let $d_{i}: \mathfrak{X} \rightarrow \mathfrak{X}$ be the $\mathbb{Q}[\beta]$-linear operator defined by

$$
d_{i} \cdot \lambda= \begin{cases}\lambda \cup\{\text { a box in } i \text {-th row }\}, & \text { if possible } \\ (-\beta) \cdot \lambda, & \text { otherwise }\end{cases}
$$

By seeing their actions on the basis, we can check that the operators $d_{1}, d_{2}, \ldots$ satisfy the Knuth relation:

LEMMA 6.1. We have the following commutative relations.

$$
\begin{aligned}
& d_{i} d_{k} d_{j}=d_{k} d_{i} d_{j}, \quad i \leqslant j<k, \\
& d_{j} d_{i} d_{k}=d_{j} d_{k} d_{i}, \quad i<j \leqslant k .
\end{aligned}
$$

EXAMPLE 6.2 .
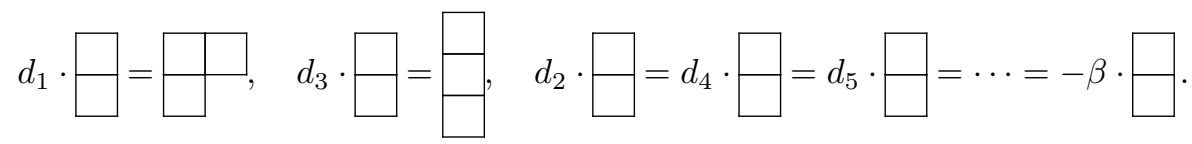

For any Young tableau $T$, we write

$$
d^{T}=d_{w_{T}(1)} d_{w_{T}(2)} \cdots d_{w_{T}(N)} .
$$

We also define the non-commutative Schur functions $s_{\lambda}\left(d_{1}, \ldots, d_{n}\right)$ in the similar manner to $s_{\lambda}\left(u_{1}, \ldots, u_{n}\right)$ (Definition 5.5).

Let $\rho: \mathfrak{X} \rightarrow \Lambda$ be the $\mathbb{Q}[\beta]$-linear map defined by

$$
\rho: \lambda \mapsto g_{\lambda}(x)
$$

The following proposition is an analogy of Proposition 5.7.

Proposition 6.3. For $\ell(\lambda) \leqslant s, \ell(\mu) \leqslant r$, we have

$$
s_{\lambda}^{(r+s-1)}(x ;-\beta) g_{\mu}(x)=\rho\left(s_{\lambda}\left(d_{1}, \ldots, d_{r+s}\right) \cdot \mu\right),
$$

where $s_{\lambda}^{(r+s-1)}(x ;-\beta)=s_{\lambda}(\overbrace{-\beta, \ldots,-\beta}^{r+s-1}, x_{1}, x_{2}, \ldots)$.

Proof. From Proposition 5.6, it suffices to prove

$$
e_{i}^{(r+s-1)}(x ;-\beta) g_{\mu}(x)=\rho\left(e_{i}\left(d_{1}, \ldots, d_{r+s}\right) \cdot \mu\right)
$$

for $0 \leqslant i \leqslant s$. Let $g(x)=\left\langle 0\left|e^{H(x)} \psi_{n_{1}-1} e^{-\theta} \cdots \psi_{n_{r}-r} e^{-\theta} \psi_{-r-1} e^{-\theta} \cdots \psi_{-r-s}\right|-r-s\right\rangle$ for any sequence of integers $n_{1}, \ldots, n_{r}$. Since $\left[a_{-i}, e^{-\theta}\right]=-(-\beta)^{i} e^{-\theta}$, we have

$$
\begin{aligned}
& p_{i}(x) g(x) \\
&=\left\langle 0\left|e^{H(x)} a_{-i} \psi_{n_{1}-1} e^{-\theta} \cdots \psi_{n_{r}-r} e^{-\theta} \cdots \psi_{-r-s}\right|-r-s\right\rangle \\
&= \sum_{j=1}^{r+s}\left\{\left\langle 0\left|e^{H(x)} \psi_{n_{1}-1} e^{-\theta} \cdots \psi_{n_{j}-j+i} e^{-\theta} \cdots \psi_{-r-s}\right|-r-s\right\rangle\right\} \\
&-(r+s-1)(-\beta)^{i} g(x)+\left\langle 0\left|e^{H(x)} \psi_{n_{1}-1} e^{-\theta} \cdots \psi_{n_{r}-r} e^{-\theta} \cdots \psi_{-r-s} a_{-i}\right|-r-s\right\rangle .
\end{aligned}
$$

Note that the last term of this expression must vanish if $i \leqslant s$. Moreover, because

$$
p_{i}^{(r+s-1)}(x ;-\beta)=p_{i} \overbrace{-\beta, \ldots,-\beta}^{r+s-1}, x)=\overbrace{(-\beta)^{i}+\cdots+(-\beta)^{i}}^{r+s-1}+x_{1}^{i}+x_{2}^{i}+\cdots,
$$

the equation can be simplified as

$$
p_{i}^{(r+s-1)}(x ;-\beta) g(x)=\sum_{j=1}^{r+s}\left\langle 0\left|e^{H(x)} \psi_{n_{1}-1} e^{-\theta} \cdots \psi_{n_{j}-j+i} e^{-\theta} \cdots \psi_{-r-s}\right|-r-s\right\rangle .
$$


From this, we find that the product $e_{i}^{(r+s-1)}(x ;-\beta) g(x)$ satisfies the following equation:

$$
\begin{aligned}
& e_{i}^{(r+s-1)}(x ;-\beta) g(x) \\
= & \sum_{1 \leqslant m_{1}<\cdots<m_{i} \leqslant r+s}\left\langle 0\left|e^{H(x)} \psi_{n_{1}-1} e^{-\theta} \cdots \psi_{n_{m_{1}}-m_{1}+1} e^{-\theta} \cdots \psi_{n_{m_{i}}-m_{i}+1} e^{-\theta} \cdots \psi_{-r-s}\right|-r-s\right\rangle .
\end{aligned}
$$

Substituting $g(x)=g_{\mu}(x)$ to this equation gives $(26)$.

ExAMPLE 6.4. Let $s=1$ and $r=0$. In this case $s_{(n)}^{(0)}(x ;-\beta)=h_{n}(x)$. Since $h_{n}\left(d_{1}\right) \cdot \varnothing=$ $d_{1}^{n} \cdot \varnothing=(n)$, we have $h_{n}(x)=g_{(n)}(x)$.

EXAMPLE 6.5 . Let $s=n$ and $r=0$. In this case $s_{\left(1^{n}\right)}^{(n-1)}(x ;-\beta)=e_{n}^{(n-1)}(x ;-\beta)=$ $\sum_{j=0}^{n}(-\beta)^{j}\left(\begin{array}{c}n-1 \\ j\end{array}\right) e_{n-j}(x)$. Since $e_{n}\left(d_{1}, \ldots, d_{n}\right) \cdot \varnothing=d_{n} \cdots d_{2} d_{1} \cdot \varnothing=\left(1^{n}\right)$, we have $\sum_{j=0}^{n}(-\beta)^{j}\left(\begin{array}{c}n-1 \\ j\end{array}\right) e_{n-j}(x)=g_{\left(1^{n}\right)}(x)$.

6.1. PIeRI TYPe FORMula FOR $e_{i}(x) g_{\lambda}(x)$. Proposition 6.3 is unfortunately a bit complicated as it contains a function of the form $s_{\lambda}^{(r+s-1)}(x ;-\beta)$. However, when the partition $\lambda$ is relatively simple (for example, if $\lambda=\left(1^{i}\right)$ or $\lambda=(i)$ ), we can handle the situation. In the following, we consider the expansions of symmetric functions of the form $e_{i}(x) g_{\lambda}(x)$ and $h_{i}(x) g_{\lambda}(x)$.

To calculate the product $e_{i}(x) g_{\lambda}(x)$, it is convenient to introduce the formal power series $E(t)=\sum_{i=0}^{\infty} e_{i}(x) t^{i}=\prod_{j=1}^{\infty}\left(1+x_{j} t\right)$. From the expression $e_{i}^{(p)}(x ;-\beta)=$ $e_{i}(\overbrace{-\beta, \ldots,-\beta}^{p}, x_{1}, x_{2}, \ldots)$, we find

$$
\sum_{i=0}^{\infty} e_{i}^{(p)}(x ;-\beta) t^{i}=(1-\beta t)^{p} E(t) .
$$

Therefore, from Proposition 6.3, we have

$$
E(t) g_{\lambda}(x) \equiv \rho\left((1-\beta t) \cdot\left(\frac{1+d_{r+s} t}{1-\beta t} \cdots \frac{1+d_{2} t}{1-\beta t} \cdot \frac{1+d_{1} t}{1-\beta t}\right) \cdot \lambda\right) \quad \bmod t^{s+1}
$$

for $\ell(\lambda) \leqslant r$. Taking the limit as $r, s \rightarrow \infty$ gives the formal expression

$$
E(t) g_{\lambda}(x)=\rho\left((1-\beta t) \cdot\left(\cdots \frac{1+d_{2} t}{1-\beta t} \cdot \frac{1+d_{1} t}{1-\beta t}\right) \cdot \lambda\right)
$$

For example, since

$$
\begin{gathered}
(1-\beta t) \cdot\left(\cdots \frac{1+d_{3} t}{1-\beta t} \cdot \frac{1+d_{2} t}{1-\beta t} \cdot \frac{1+d_{1} t}{1-\beta t}\right) \cdot \varnothing=\varnothing+\square \frac{t}{1-\beta t}+\square \frac{t^{2}}{(1-\beta t)^{2}}+\cdots, \\
(1-\beta t) \cdot\left(\cdots \frac{1+d_{3} t}{1-\beta t} \cdot \frac{1+d_{2} t}{1-\beta t} \cdot \frac{1+d_{1} t}{1-\beta t}\right) \cdot \square \\
=\left(\square \frac{1}{1-\beta t}+\square \frac{t}{(1-\beta t)^{2}}+\square \frac{t^{2}}{(1-\beta t)^{3}}+\cdots\right) \\
+\left(\square \frac{t}{1-\beta t}+\square \frac{t^{2}}{(1-\beta t)^{2}}+\frac{t^{3}}{\square} \frac{\square}{(1-\beta t)^{3}}+\cdots\right),
\end{gathered}
$$


we have

$$
\begin{aligned}
& E(t)=g_{\varnothing}+g_{\square} t+\left(\beta g_{\square}+g_{\square}\right) t^{2}+\left(\beta^{2} g_{\square}+2 \beta g g_{\square}+g_{\square}\right) t^{3}+\cdots, \\
& E(t) g_{\square}=g_{\square}+\left(\beta g_{\square}+g_{\square}+g_{\square}{ }_{\square}\right) t \\
& +\left(\beta^{2} g_{\square}+2 \beta g \square+\beta g \square+g \square+g \square \square_{\square}+t^{2}+\cdots\right.
\end{aligned}
$$

6.2. Pieri type Formula For $h_{i}(x) g_{\lambda}(x)$. Let $H(t):=\sum_{i=0}^{\infty} h_{i}(x) t^{i}=\prod_{j=1}^{\infty} \frac{1}{1-x_{i} t}$ be the generating function of $h_{i}(x)$. Since $h_{i}^{(p)}(x ;-\beta)=h_{i} \overbrace{-\beta, \ldots,-\beta}^{p}, x_{1}, x_{2}, \ldots)$, we have

$$
\sum_{i=0}^{\infty} h_{i}^{(p)}(x ;-\beta) t^{i}=(1+\beta t)^{-p} H(t) .
$$

Therefore, from Proposition 6.3, we have

$$
H(t) g_{\lambda}(x)=\rho\left(\frac{1}{1+\beta t} \frac{1+\beta t}{1-d_{1} t} \frac{1+\beta t}{1-d_{2} t} \cdots \lambda\right),
$$

where $\frac{1}{1-d_{i} t}=1+d_{i} t+d_{i}^{2} t^{2}+\cdots$. We note that the expression (29) does not cause a confusion because $\frac{1+\beta t}{1-d_{i} t} \cdot \lambda=\lambda$ for $i>\ell(\lambda)+1$. For example, we have

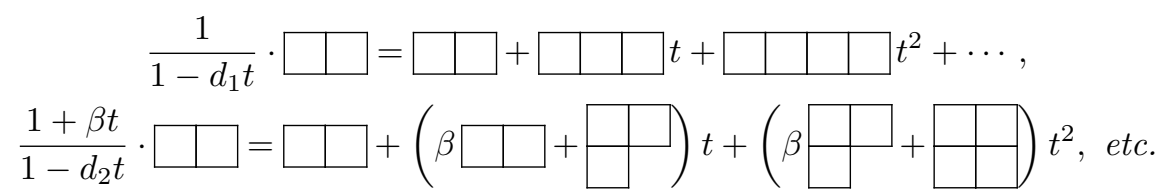

Therefore we obtain

$$
\begin{aligned}
& H(t)=g_{\varnothing}+g_{\square} t+g_{\square \square} t^{2}+g_{\square \square} t^{3}+\cdots, \\
& H(t) g_{\square}=g_{\square}+\left(\beta g_{\square}+g_{\square \square}+g_{\square}\right) t+\left(\beta g_{\square \square}+g_{\square \square}+g_{\square}{ }_{\square}\right) t^{2}+\cdots, \\
& H(t) g_{\square \square}=g_{\square \square}+\left(\beta g_{\square \square}+g_{\square \square}+g_{\square \square \square}\right) t
\end{aligned}
$$

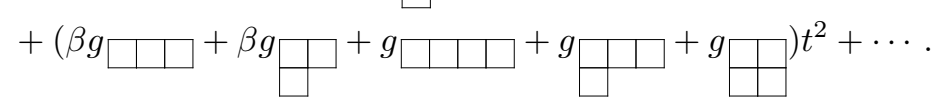

REFERENCES

[1] Alexander Alexandrov and Anton Zabrodin, Free fermions and tau-functions, J. Geom. Phys. 67 (2013), 37-80.

[2] Anders S. Buch, A Littlewood-Richardson rule for the K-theory of Grassmannians, Acta Math. 189 (2002), no. $1,37-78$.

[3] Sergey Fomin and Curtis Greene, Noncommutative Schur functions and their applications, Discrete Math. 193 (1998), no. 1-3, 179-200.

[4] Sergey Fomin and Anatol N. Kirillov, Grothendieck polynomials and the Yang-Baxter equation, Proc. Formal Power Series and Alg. Comb, 1994, pp. 183-190.

[5] William Fulton, Young tableaux: With applications to representation theory and geometry, London Mathematical Society Student Texts, Cambridge University Press, 1996.

[6] Thomas Hudson, Takeshi Ikeda, Tomoo Matsumura, and Hiroshi Naruse, Degeneracy loci classes in K-theory - determinantal and Pfaffian formula, Adv. Math. 320 (2017), 115-156.

[7] Takeshi Ikeda and Hiroshi Naruse, $K$-theoretic analogues of factorial Schur $P$ - and Q-functions, Adv. Math. 243 (2013), 22-66.

[8] Shinsuke Iwao and Hidetomo Nagai, The discrete Toda equation revisited: dual $\beta$-Grothendieck polynomials, ultradiscretization, and static solitons, J. Phys. A 51 (2018), no. 13, 134002. 
[9] Michio Jimbo and Tetsuji Miwa, Solitons and infinite-dimensional Lie algebras, Publ. Res. Inst. Math. Sci. 19 (1983), no. 3, 943-1001.

[10] Victor G. Kac, Ashok K. Raina, and Natasha Rozhkovskaya, Bombay lectures on highest weight representations of infinite dimensional Lie algebras, Advanced Series in Mathematical Physics, vol. 29, World scientific, 2013.

[11] Anatol N. Kirillov, On some quadratic algebras I $\frac{1}{2}$ : combinatorics of Dunkl and Gaudin elements, Schubert, Grothendieck, Fuss-Catalan, universal Tutte and reduced polynomials, SIGMA Symmetry Integrability Geom. Methods Appl. 12 (2016), Paper no. 002 (172 pages).

[12] Alain Lascoux and Hiroshi Naruse, Finite sum Cauchy identity for dual Grothendieck polynomials, Proc. Japan Acad. Ser. A Math. Sci. 90 (2014), no. 7, 87-91.

[13] Alain Lascoux and Marcel-Paul Schützenberger, Structure de Hopf de l'anneau de cohomologie et de l'anneau de Grothendieck d'une variété de drapeaux, C. R. Acad. Sci. Paris Sér. I Math. 295 (1982), no. 11, 629-633.

[14] _ Symmetry and flag manifolds, Invariant theory (Montecatini, 1982), Lecture Notes in Math., vol. 996, Springer, Berlin, 1983, pp. 118-144.

[15] Cristian Lenart, Combinatorial aspects of the K-theory of Grassmannians, Ann. Comb. 4 (2000), no. 1, 67-82.

[16] Ian G. Macdonald, Symmetric functions and Hall polynomials, Oxford university press, 1998.

[17] Tetsuji Miwa, Michio Jimbo, Etsuro Date, and Miles Reid, Solitons: Differential equations, symmetries and infinite dimensional algebras, Cambridge Tracts in Mathematics, vol. 135, Cambridge University Press, 2012.

[18] Kohei Motegi and Kazumitsu Sakai, Vertex models, TASEP and Grothendieck polynomials, J. Phys. A 46 (2013), no. 35, 355201, 26.

[19] _ K-theoretic boson-fermion correspondence and melting crystals, J. Phys. A 47 (2014), no. $44,445202,30$

[20] Masaki Nakagawa and Hiroshi Naruse, Universal factorial Schur P, Q-functions and their duals, https://arxiv.org/abs/1812.03328, 2018.

[21] Mark Shimozono and Mike Zabrocki, Stable Grothendieck polynomials and $\Omega$-calculus, unpublished, 2011.

[22] Damir Yeliussizov, Duality and deformations of stable Grothendieck polynomials, J. Algebraic Combin. 45 (2017), no. 1, 295-344.

Shinsuke Iwao, Department of Mathematics, Tokai University, 4-1-1, Kitakaname, Hiratsuka, Kanagawa 259-1292, Japan.

E-mail : iwao@tokai.ac.jp 